\title{
Mieux connaître le devenir biologique des futurs matériaux nucléaires, une nécessité pour maintenir un bon niveau de radioprotection
}

\author{
H. MÉTIVIER ${ }^{1}$, R. GUILLAUMONT ${ }^{2}$
}

(Manuscrit reçu le $1^{\text {er }}$ janvier 2007, accepté le 26 février 2007)

Résumé

\begin{abstract}
Un important programme de recherches, appelé « Generation IV », est actuellement développé au niveau international pour préparer les réacteurs du futur et leurs « cycle de combustible». Son objectif est de satisfaire les besoins croissants en énergie électrique dans le cadre d'un développement durable. L'un des aspects majeurs de ce programme est la recherche et le développement de nouveaux matériaux nucléaires fissiles/fertiles. Malheureusement, on s'aperçoit aujourd'hui que, pour de nombreux composés candidats pour développer ces matériaux, il n'existe pas de données radiotoxicologiques permettant au médecin du travail d'évaluer le risque réel en cas de contamination et de prescrire un traitement de décontamination adapté. Il conviendrait donc de relancer d'urgence les études de poste où sont, ou bien seront, mis en ouvre ces composés ou matériaux. L'expérience passée a montré que la prévision en matière de radioprotection était difficile et qu'une validation expérimentale du comportement des éléments lourds radiotoxiques ou toxiques in vitro et in vivo était nécessaire.
\end{abstract}

Abstract To better know the biological behaviour of future nuclear materials: a need to maintain a good level of protection against ionising radiation.

An important program of research, called "Generation IV" is currently developed at an international level to prepare the launching of reactors for the future. Its objective is to satisfy the growing needs in electric power within the framework for a sustainable development. One of the major aspects of this program is the search and the development for new nuclear materials containing fissile/fertile isotopes. Unfortunately, one realizes today that for many compounds considered to be used to manufacture these materials, there are no radiotoxicologic data, allowing to the occupational physicist to evaluate the real risk in the event of contaminations and to prescribe adapted therapeutic de-corporation. An emergency revival of "workplace" studies, where are or will be handled these compounds or materials is mandatory. The lesson of the past showed that the prevision in radioprotection was rather difficult and that an experimental validation of the behaviour of hazardous heavy elements both in vitro and in vivo was necessary.

Keywords: nuclear fuels / generation IV / internal dosimetry / biokinetics / DPUI

${ }^{1} 2$ allée des Hautes Futaies, 91450 Soisy-sur-Seine, France.

${ }^{2}$ Membre de l'Académie des sciences, 7 rue E. Branly, 91120 Palaiseau, France. 


\section{Introduction}

La définition de «systèmes nucléaires » pour le futur, réacteurs et installations associées du cycle du combustible, s'est essentiellement faite avec l'objectif de satisfaire des besoins croissants en énergie électrique dans le cadre d'un développement durable. Plusieurs pays se sont ainsi associés dans un consortium couramment appelé «Generation $I V »^{3}$ en vue de développer des nouveaux réacteurs répondant à plusieurs critères. Le premier est celui de l'économie des ressources naturelles en matières nucléaires fissiles et fertiles. Les deux autres sont le respect de l'environnement et la minimisation de la production de déchets « radiotoxiques à long terme », c'est-à-dire contenant des radionucléides à vie longue (CEA, 2005). L'aspect économique, la sûreté et la fiabilité, ainsi que la limitation des risques de prolifération sont évidemment les autres critères associés au développement de ces nouveaux systèmes, déjà mis en pratique aujourd'hui.

L'un des aspects majeurs des projets est, du point de vue du chimisteradiochimiste et du radioprotectionniste, le développement de nouveaux matériaux nucléaires fissiles/fertiles. Ceux-ci font d'ailleurs, dès aujourd'hui, l'objet de recherches et développements dans une dizaine de pays du monde. Ces travaux exposent ainsi des chercheurs au risque de contamination accidentelle par des composés radioactifs souvent nouveaux. Dans un tel cas, la démarche usuelle, pour le radioprotectionniste et le médecin du travail, est de calculer la dose reçue pour évaluer le risque encouru et ensuite d'envisager un éventuel traitement. Cela nécessite de bien connaître le devenir biologique des produits contaminants qui pourraient provenir de ces matériaux. On peut faire des prévisions par analogies chimiques mais une validation expérimentale est nécessaire. Les données que l'on pourrait ainsi obtenir aideraient les médecins du travail dans la gestion de risques radiologiques nouveaux. Si les systèmes nucléaires de « Generation $I V$ » voient le jour, les problèmes seront d'une autre ampleur en raison du nombre de personnes qui seront impliquées et des quantités importantes de différentes matières radioactives mises en jeu. Cela renforce évidemment la nécessité d'action que l'on vient de souligner. Bien que la maturité industrielle des systèmes nucléaires du futur ne soit pas attendue avant quelques décennies, on sait que dans le nucléaire les échéances doivent être anticipées très longtemps en avant des dates annoncées.

\section{L'évaluation du risque de contamination interne}

La Commission internationale de protection radiologique (CIPR) a très tôt souligné l'importance du rôle de la physicochimie d'un radionucléide dans son

\footnotetext{
${ }^{3}$ Generation IV est un forum international regroupant la France, le Royaume-Uni, les états européens du traité Euratom, la Suisse, le Japon, la Corée du Sud, l'Afrique du Sud, l'Argentine, le Brésil, les États-Unis et le Canada. On garde ici l'appellation abrégée internationale pour Forum international Generation IV.
} 
comportement biologique. Cet aspect des études de radioprotection est capital pour l'évaluation des différents facteurs de transfert du radionucléide à l'homme et par conséquent pour le calcul de la dose. Il relève de nombreux paramètres.

Pour les radionucléides dont le temps de séjour dans l'organisme est long, comme les isotopes de plutonium et d'uranium, et d'une façon générale les isotopes des actinides, la nature physicochimique de la forme incorporée à l'organisme est essentielle pour le calcul de la dose délivrée au travailleur contaminé.

Très tôt, les laboratoires spécialisés dans les effets de la contamination interne des radionucléides ont étudié, à la demande des opérateurs industriels et des laboratoires de recherches, le devenir biologique des composés les plus divers utilisés dans l'industrie nucléaire. À partir de ces données, des modèles dosimétriques ont été bâtis qui permettent d'évaluer les doses reçues par les travailleurs et de surveiller que les limites réglementaires fixées par les autorités sont bien respectées.

Plus récemment la CIPR (ICRP, 1995a) a recommandé de faire des études spécifiques à chaque "poste de travail», permettant ainsi, en cas de contamination, un calcul réaliste et adapté de la dose reçue, et par conséquent une évaluation plus précise du risque (Stradling et al., 1978; Hengé-Napoli et al., 1994 ; Moody et al., 1994 ; Ansoborlo et al., 2002, 1995). Ce travail ne peut se faire que s'il y a une collaboration étroite entre les différents acteurs impliqués dans la radioprotection. L'opérateur doit mettre à la disposition du chercheur toutes les informations qu'il possède pour mieux appréhender le devenir biologique des produits radioactifs contaminants connus ou pressentis. Il doit également en informer les services de radioprotection mais aussi les services médicaux du travail. Ce n'est qu'à ce prix que le médecin pourra faire une évaluation correcte du risque encouru par le travailleur. Ce système donne toute satisfaction dans l'industrie nucléaire actuelle.

Malgré ce résultat satisfaisant, et contrairement à ce que l'on pouvait penser il y a encore quelques années, le besoin d'informations nouvelles demeure et plus particulièrement depuis la relance des recherches concernant de futurs systèmes nucléaires qui devraient, à terme, remplacer les réacteurs et le cycle du combustible uranium/plutonium actuels.

Fort curieusement, si la recherche radiotoxicologique et toxicologique a toujours accompagné avec succès la recherche technologique en matière d'énergie nucléaire (Métivier et Masse, 1986), on observe aujourd'hui une rupture entre l'avance de la recherche technologique et la recherche dans ces domaines (Métivier, 1989). 
Dans cet article, on examine la situation du devenir biologique de nouveaux composés en cours d'étude pour lesquels on ne dispose d'aucune information directe. Certes on peut faire quelques prévisions mais l'expérience a montré, maintes fois, que si la prévision est nécessaire, la vérification expérimentale est incontournable. On a de multiple exemples avec le tributylphophate de plutonium (Métivier et al., 1983, 1989), le tétrafluorure d'uranium (André et al., 1989a, 1989b), différents alliages du béryllium (André et al., 1987) et différents oxydes de plutonium (Mewhinney et al., 1976 ; Métivier, 1979 ; Bair et al., 1989, 1980 ; Morgan et al., 1988 ; Sato et al., 1999). Il convient donc d'envisager de nouvelles recherches expérimentales en radiotoxicologie.

\section{Rappel sur les modèles dosimétriques de la CIPR}

Les modèles utilisés par la majorité des services médicaux en charge de la protection des travailleurs ont été développés par le comité 2 de la CIPR. Ces modèles sont de plusieurs types. Les modèles biocinétiques décrivent le devenir biologique des radionucléides. On les appelle «modèles d'entrée » lorsqu'ils permettent de prévoir le devenir biologique à partir d'une source d'entrée, inhalation, ingestion ou blessure. On les appelle «modèles systémiques » lorsqu'ils décrivent la répartition du radionucléide à partir du sang, et son élimination par les voies naturelles, une fois le radionucléide transféré de la porte d'entrée au compartiment sanguin. Enfin, des «modèles dosimétriques » permettent de calculer la dose d'irradiation en fonction du temps pour un organe contaminé, mais aussi, lorsque c'est nécessaire, la dose d'irradiation d'un organe en fonction du temps par les autres organes contaminés, agissant alors comme termes sources.

La CIPR a développé deux modèles d'entrée, le modèle respiratoire appelé «Human Respiratory Tract Model» (HRTM) (ICRP, 1994a) et le modèle alimentaire «Human Alimentary Tract Model» (HATM) (ICRP, 2007). Le troisième, « le modèle blessure » est en cours d'élaboration par le comité américain de radioprotection (NCRP) (Breitenstein et al., 2007).

Les modèles d'entrée sont très étroitement associés à la forme physicochimique du polluant radioactif, taille et nature des poussières inhalées, nature du composé ingéré ou injecté par blessure. À l'inverse, une fois solubilisé ou transformé sur le site d'entrée, poumon, tube digestif ou site de la blessure, le radionucléide transféré dans le compartiment sanguin se répartit dans l'organisme selon la forme physicochimique présente dans le sang et non plus selon la nature du polluant d'origine. Ces derniers modèles sont dessinés par familles chimiques de contaminants, présentant en général de grandes similarités pour la distribution dans les organes des éléments qu'ils renferment. 


\subsection{Le modèle respiratoire}

Le modèle respiratoire divise le système respiratoire en 5 régions, (1) le compartiment extra-thoracique (ET) lui-même divisé en deux parties ET1, antérieure au nez et ET2 qui comprend la partie postérieure au nez, la voie orale, le pharynx et le larynx, (2) la région thoracique (BB) comporte la trachée, les grosses bronches qui correspondent aux huit premières générations des voies respiratoires, (3) la partie bronchiolaire comporte les générations 9 à 15 des bronchioles, (4) la partie alvéolo-interstitielle (AI) est la zone d'échange gazeuse du poumon, (5) les tissus ou nodules lymphatiques sont associés aux parties extrathoraciques, ils sont appelés $\mathrm{LN}_{\mathrm{ET}}$ et $\mathrm{LN}_{\mathrm{TH}}$ respectivement. Cette dernière région n'est pas affectée lors du dépôt direct des particules inhalées.

La quantité de polluant inhalé déposée dans le poumon et sa localisation dépend de la taille aérodynamique des particules inhalées. La taille aérodynamique est une combinaison de la taille réelle et de la densité de la particule. La cinétique d'élimination du polluant à partir du dépôt dans le poumon est liée à sa nature physicochimique, notamment à la solubilité des particules qui dépend de la taille (voir § 5.2.). Le modèle propose une classification par défaut de tous les composés et attribue à chaque classe des paramètres cinétiques par défaut. Ces classes sont respectivement la classe F pour les composés très solubles (Fastly soluble), M pour les composés moyennement solubles (Moderatly soluble) et S pour les composés dits insolubles (Slowly soluble). La solubilité à laquelle on se réfère est la solubilité in vitro quasi instantanée des composés dans des milieux artificiels proches des milieux physiologiques (voir $§ 3.4$.).

À partir de ce modèle, on établit des coefficients de dose par unité d'incorporation (DPUI) pour l'inhalation, exprimés en Sv par Bq inhalé. Ils sont publiés dans les textes réglementaires. Si les services de protection connaissent les caractéristiques spécifiques des polluants, ils pourront substituer les paramètres par défaut du modèle par des paramètres spécifiques. Les coefficients de dose calculés ainsi sont appelés «Dose par unité d'incorporation spécifique ». Le modèle permet aussi au médecin du travail d'adapter ses calculs de dose au profil individuel du travailleur, son tabagisme par exemple.

La qualité de la protection des travailleurs ou l'efficacité des traitements mis en place dépend étroitement de la bonne connaissance des caractéristiques du polluant inhalé. Seule le permet une collaboration étroite entre les opérateurs et concepteurs d'une part, et les services de surveillance de radioprotection d'autre part. 


\subsection{Le modèle alimentaire}

Un nouveau modèle alimentaire (HATM) vient d'être mis en place par la CIPR (ICRP, 2007). Ce modèle est étroitement lié à l'anatomie du système alimentaire. Il est divisé en plusieurs régions ; la bouche, l'œsophage, l'estomac, l'intestin grêle, le gros intestin divisé en partie gauche du colon, partie droite du colon et un dernier compartiment regroupant la partie sigmoïde du colon et le rectum. Le modèle prend en compte la rétention potentielle au niveau de plusieurs compartiments, les dents, la muqueuse buccale, l'estomac et l'intestin dans son ensemble. Ce modèle considère que le transfert vers le sang peut se faire au niveau de la bouche, de l'estomac et des différents compartiments de l'intestin. Tout comme le modèle respiratoire, le modèle alimentaire propose une classification par défaut de tous les composés et attribue à chaque classe des paramètres de transfert et de rétention. Ces classes sont respectivement la classe F pour les composés très solubles (Fastly soluble), M pour les composés moyennement solubles (Moderatly soluble) et $\mathrm{S}$ pour les composés dits insolubles (Slowly soluble). Son utilisation permet d'obtenir des DPUI pour l'ingestion.

De nouveau, lorsque l'information existe, le service de surveillance peut utiliser des paramètres spécifiques comme cela était déjà recommandé pour le modèle respiratoire.

\subsection{Le «modèle blessure »}

Ce modèle en cours d'établissement par le National Council on Radiation Protection and Measurements américain (NCRP), est également influencé par les caractéristiques physicochimiques du polluant. Comme les autres modèles de la CIPR, c'est un modèle à compartiments multiples qui décrit par des équations linéaires du premier ordre la rétention et l'élimination des radionucléides déposés à l'endroit blessé. Sept catégories par défaut ont été définies pour décrire la rétention in situ, quatre catégories pour la contamination par des composés initialement solubles, faible (Weak), modérée (Moderate), forte (Strong) et avide (Avid) et trois catégories pour des composés considérés comme initialement solides, colloïdes, particules et fragments (Breitenstein et al., 2007).

\subsection{La nature des études en radiotoxicologie}

Pour calculer les doses reçues après inhalation de radionucléides, il est nécessaire de connaitre leur solubilité telle qu'elles ont été définies par la CIPR. On peut les obtenir par retour d'expérience mais il est plus efficace de les déterminer par expérimentation animale ou bien par des tests in vitro avant même qu'une contamination ait eu lieu, ce sont les « études de postes ». 
Très tôt des tests de solubilité in vitro ont été développés (Kanapilly et al., 1973 ; Cooke et Holt, 1974 ; Eidson, 1994) utilisant pour la plupart des solutions simulant la composition chimique des liquides extracellulaires décrits par Gamble (1967). Toutefois, l'universalité de cette méthode est parfois mise en défaut compte tenu de la méthodologie appliquée (Fleischer, 1975 ; Carter, 1995). C'est le cas, par exemple lorsque la fraction solubilisée est mesurée après soutirage séquentiel de fractions de soluté. On peut observer alors que chaque fraction dissout la même quantité du composé étudié suggérant ainsi un effet de saturation dans la solution test. Pour pallier cet inconvénient des techniques de dissolution par flux continu ont été proposées. Dans ce cas, la vitesse du flux peut influencer fortement la qualité du résultat. Pour l'uranium, par exemple, la technique expérimentale est plus importante que le choix du milieu de dissolution. Ainsi l'étude in vitro de la solubilité de $\mathrm{UF}_{4}$ a montré que l'utilisation d'un fluide de référence ne permet pas à lui seul de décrire la solubilité in vivo de ce composé (André et al., 1989a, 1989b).

Des techniques cellulaires ont été également proposées (Poncy et al. 1992 ; Lirsac et al., 1989).

Il apparait donc, que quelle que soit l'aide apportée par ces tests de dissolution in vitro, il est nécessaire d'effectuer des expérimentations animales pour confirmer les résultats obtenus. Pour obtenir la classe de solubilité définie par la CIPR, une étude sur le rongeur (rat ou souris) pourra être suffisante, par contre la recherche de fonctions fiables de rétention et d'excrétion extrapolables à l'homme nécessitera l'utilisation d'espèce animale de plus grande taille, d'espérance de vie plus longue. Le chien « beagle » a été largement utilisé aux États-Unis (Thompson, 1989) et le primate en France (Masse et Métivier, 1990). Les résultats obtenus chez plusieurs espèces animales et l'homme ont fait l'objet d'une intercomparaison au niveau européen, restée à nos jours, unique (Bailey et al., 1989).

Enfin lorsque l'information est manquante ou parcellaire il est souvent procédé par analogie chimique dans l'attente d'un résultat expérimental. Des études comparatives de constantes de stabilités de complexes d'intérêt biologique ont permis à l'intérieur d'une famille chimique d'expliquer des différences de comportement (Durbin, 1960 ; Masse et al., 1973).

\section{Les recherches fondamentales et technologiques actuelles}

La relance éventuelle du nucléaire, accompagnée d'une volonté délibérée de réduire, dans les réacteurs d'aujourd'hui mais surtout dans les réacteurs du futur, la production d'actinides mineurs (neptunium, américium, curium) a conduit à entreprendre des recherches innovantes pour de nouveaux combustibles nucléaires et des cibles de transmutation. En effet, la radiotoxicité potentielle à long terme des 
déchets nucléaires est portée par le plutonium et ces actinides, qu'il conviendrait, pour la diminuer, de détruire par transmutation dans des réacteurs appropriés. Il s'agit essentiellement de combustibles et cibles pour les réacteurs à neutrons rapides, seuls bien adaptés à la transmutation de ces éléments (OCDE 2005, 2006 ; Ogawa et al., 2005 ; Pillon, 2004 ; USDOE, 2006). D'une façon générale, la distinction entre combustible et cible pour la transmutation est la suivante. On considère que le combustible est un solide dont la composition et la nature permettent la propagation de la fission tandis que les cibles sont inertes de ce point de vue. Le combustible de transmutation à base d'uranium et plutonium, jusqu'à $20 \%$ par exemple, sera peu concentré en actinides mineurs, quelques pourcents $(2,5$ à $5 \%)$, tout le contraire d'une cible de transmutation sans uranium (ni plutonium) où leur concentration pourrait atteindre 50 pourcents. Pour une quantité donnée d'actinides mineurs à transmuter, il faut évidemment mettre en œuvre beaucoup plus de « combustible » que de « cible».

Le combustible actuel, qu'il s'agisse de UOX ou de MOX, évoluera tant pour les réacteurs actuels que pour les réacteurs de troisième génération, comme l'EPR, et a fortiori pour des combustibles incorporant, ou non, des actinides mineurs que l'on souhaiterait transmuter dans des réacteurs de quatrième génération.

Tant que l'on restera, pour la préparation et l'utilisation du combustible frais, à la mise en œuvre de mélanges d'oxydes d'uranium et de plutonium il n'y aura pas, au plan radiotoxicologique, de changements qualitatifs majeurs par rapport à la situation actuelle. En effet, seules les compositions isotopiques du combustible nucléaire neuf et usé pourront varier mais leurs propriétés chimiques, dans toutes les circonstances où ils pourraient être manipulé intact ou altéré et donner lieu à des expositions ou des émissions de produits radioactifs, resteront les mêmes. Encore que de hauts taux de combustion pourraient provoquer de façon mineure l'apparition de phases chimiques nouvelles. Ce «non changement» est aussi valable aux étapes de préparation du combustible, voire de son retraitement, qui resteront ce qu'elles sont à quelques aménagements près.

Il en sera autrement avec les nouveaux combustibles ou les cibles de transmutation pour les réacteurs de quatrième génération (voir ci-dessous) et, à côté des recherches physicochimiques entreprises il paraît donc indispensable, comme on l'a dit, de conduire des études toxicologiques complémentaires à celles qui ont été conduites sur les systèmes nucléaires actuels. Elles devraient porter tant sur le rôle des actinides en général que sur les composés solides d'actinides nouveaux et sur les nouvelles solutions liquides de ces éléments qui pourraient être introduits dans la chaîne industrielle, allant du retraitement du combustible usé, ou bien des cibles, à la préparation du combustible et de cibles de deuxième génération (voir de troisième). Ces opérations sur une matière de plus en plus 
radioactive sont nécessaires pour extraire les actinides, d'une part du combustible usé classique et d'autre part des combustibles de transmutation, voire de cibles, car la disparition des radionucléides par transmutation ne pourra vraisemblablement pas se faire en une seule passe en réacteurs de transmutation. En mode hétérogène, c'est-à-dire en utilisant des cibles, on pourrait obtenir $90 \%$ de transmutation ne justifiant pas un traitement ultérieur, mais il faudrait au moins une décennie d'irradiation. Par ailleurs, il faudrait aussi examiner si on dispose ou non d'informations concernant le devenir biologique de nouvelles molécules utilisées pour séparer les actinides, qui pourraient avoir une toxicité chimique tout en véhiculant les actinides. À cet égard, on notera les hésitations passées à propos du tributylphosphate de plutonium ou du complexe de plutonium avec la trilaurylamine (Métivier et al., 1983, 1989 ; Nolibé et al., 1989).

On n'examine pas ici l'impact radiologique par exposition directe aux rayonnements qui pourrait être dû au recyclage des actinides lequel conduit à une augmentation considérable de rayonnement gamma, voire neutronique, émis par la matière radioactive mise en jeu.

Les différents concepts retenus pour les systèmes de « Generation $I V$ » sont les suivants (CEA, 2005) :

- le SFR (réacteur à neutrons rapides refroidi au sodium) est associé à un cycle fermé permettant le recyclage de l'ensemble des actinides en mode homogène ou hétérogène et la régénération du plutonium. Il utiliserait soit un combustible métallique soit un combustible MOX (mode homogène) soit des cibles (mode hétérogène). Des réacteurs de ce type, mais non transmuteurs d'actinides mineurs, ont déjà fait l'objet de développements industriels ;

- le LFR (réacteur à neutrons rapides refroidi au plomb) est associé à un cycle fermé pour une utilisation optimale de l'uranium. Il utiliserait soit un combustible métallique soit un combustible nitrure ;

- le SCWR (réacteur à neutrons thermiques modéré et refroidi par de l'eau supercritique) est associé à un cycle ouvert. Il utiliserait un combustible MOX ;

- le VHTR (réacteur à neutrons thermiques à très haute température refroidi à l'hélium) est associé à un cycle ouvert. Il serait couplé avec une installation de fabrication d'hydrogène. Il utiliserait un combustible MOX particulier à base de particules en raison de l'emploi d'un caloporteur gaz ;

- le GFR (réacteur à neutrons rapides refroidi à l'hélium) est associé à un cycle fermé permettant le recyclage de l'ensemble des actinides en mode homogène avec un gain de régénération de plutonium supérieur à 1 . Il utiliserait un combustible MOX particulier à base de particules ;

- le MSR (réacteur à neutrons épithermiques modéré au graphite et refroidi par des sels fondus) est associé à un cycle fermé avec retraitement continu in situ. Le combustible/cible serait une solution de fluorures de thorium et d'actinides. 
En complément de ces concepts de réacteurs, il existe le concept de système ADS (Accelerator Driven System). Il s'agirait de réacteurs à neutrons rapides souscritiques dédiés à la transmutation des actinides mineurs en mode hétérogène, les neutrons étant fournis aux réacteurs par spallation avec un faisceau de proton de haute énergie.

Tous ces réacteurs fonctionneraient à des températures nettement supérieures à celle des réacteurs actuels.

Les efforts semblent se concentrer aujourd'hui sur le SFR et le VHTR, ce dernier ayant comme prolongement le GFR. Le concept ADS est étudié au niveau européen. Le MSR est associé à la filière thorium et de ce point de vue apparaît comme une rupture dans le nucléaire actuel. Aussi son développement, voire son évolution vers un spectre de neutrons rapides, nécessite-t-il plus d'études que les innovations dans des filières à uranium/plutonium. Le radioélément prioritaire à transmuter, outre le plutonium, est l'américium. Le curium pose d'énormes difficultés pratiques. En France vers 2020 un prototype de SFR et un réacteur expérimental de GFR pourraient exister, mais rien n'est encore décidé.

À côté du combustible oxyde largement utilisé sous forme de pastilles (voire de microbilles dans certains assemblages MOX pour réacteurs à neutrons rapides) on voit apparaître pour de futurs combustibles les possibilités d'utiliser des carbures et nitrures d'actinides et on voit aussi apparâtre la possibilité d'utiliser deux nouveaux types de combustible, le combustible à particules enrobées et le combustible liquide à sels fondus. Quant au combustible oxyde, il pourrait être largement différent de ceux utilisés jusqu'à aujourd'hui (voir § 5.2.). Le combustible à particules pour réacteurs refroidis par un gaz a déjà été expérimenté sur des réacteurs prototypes. Il est fait de particules micrométriques de céramique dense et réfractaire, recouvertes de plusieurs couches de carbone et par exemple d'une couche de carbure de silicium, dont le rôle est de confiner les produits de fission et les actinides, comme le fait la gaine des aiguilles de combustible UOX et MOX actuelles. Des réacteurs à sels fondus ont aussi été expérimentés.

Les cibles de transmutation n'existent pas aujourd'hui en tant qu'objet industriel. Compte tenu de leur teneur élevée en actinides, il s'agira toujours d'objets à composés chimiques très particuliers.

De très nombreux composés ont été préparés pour étudier leur comportement sous irradiation en réacteurs en vue de mettre au point soit les combustibles du futur soit des cibles de transmutation. On indique dans le tableau I les principales familles de composés avec quelques exemples. 


\section{TABLEAU I}

Principales familles de composés avec quelques exemples. Les informations proviennent pour la majorité d'entre elles des deux congrès « GLOBAL » (GLOBAL, 2003, 2005) et de Pillon (2004). Main families of compounds with some examples. Information come for the majority of them from the two GLOBAL conferences (GLOBAL, 2003, 2005) and from Pillon (2004).

\begin{tabular}{|c|c|}
\hline Famille & Composés chimiques d'actinides \\
\hline \multirow[t]{3}{*}{ Oxydes } & 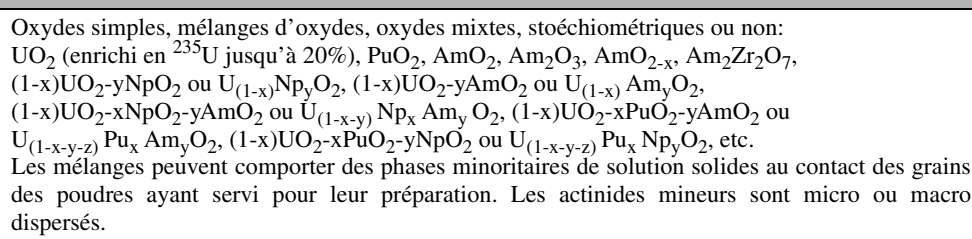 \\
\hline & $\begin{array}{l}\text { Solutions solides d'oxydes d'actinides: } \\
\mathrm{Zr}_{(1-2 \mathrm{x}-\mathrm{y})} \mathrm{M}_{\mathrm{y}} \mathrm{Y}_{2 \mathrm{x}} \mathrm{O}_{(2-\mathrm{x})} \text { avec } \mathrm{M}=\mathrm{Pu} \text {, Am et zircone stabilisée à l'yttrium. Les composés avec Am } \\
\text { sont souvent non-stoéchiométriques (ils renferment alors des ions } \mathrm{Am}^{3+} \text { et } \mathrm{Am}^{4+} \text { ) }\end{array}$ \\
\hline & $\begin{array}{l}\text { Solutions solides d'oxydes d'actinides non-stoéchiométriques : } \\
\mathrm{U}_{(1-\mathrm{x}-\mathrm{y})} \mathrm{Am}_{2 \mathrm{yz}}{ }^{3+} \mathrm{Am}_{\mathrm{y}(1-2 \mathrm{z})}{ }^{4+} \mathrm{Np}_{\mathrm{x}} \mathrm{O}_{2-\mathrm{z}},(\mathrm{Pu}, \mathrm{Am}, \mathrm{Cm}, \mathrm{Zr}) \mathrm{O}_{2-\mathrm{X}},(\mathrm{Pu}, \mathrm{Am}, \mathrm{Cm}, \mathrm{Th}) \mathrm{O}_{2-\mathrm{X}}\end{array}$ \\
\hline Métaux & $\begin{array}{l}\text { Alliages métalliques à base de } \mathrm{Zr} \text { : } \\
\text { UPuZr, UPuZr/Np/Am/Cm, PuZr, Pu/Zr/Np/Am/Cm. }\end{array}$ \\
\hline Carbures & $(\mathrm{U}, \mathrm{Pu}) \mathrm{C}, \mathrm{UPuC}$, Carbure dans une matrice $\mathrm{SiC}$ et $\mathrm{Zr}_{3} \mathrm{Si}_{2}$ \\
\hline Nitrures & $\begin{array}{l}\mathrm{UN}, \mathrm{PuN}, \text { nitrures a base de } \mathrm{ZrN} \text { : } \\
(\mathrm{ZrM}) \mathrm{N} \text { avec } \mathrm{M}=\mathrm{Pu}, \mathrm{Np}, \mathrm{Am} \text { et à base d'autres nitrures, } \mathrm{Hf} \text { et } \mathrm{Y}\end{array}$ \\
\hline Fluorures & $\mathrm{UF}_{4}, \mathrm{ThF}_{4}$ et $\mathrm{UF}_{4}$ dans LiF-BeF${ }_{2}, \mathrm{PuF}_{3}, \mathrm{NpF}_{3}, \mathrm{AmF}_{3}$ \\
\hline \multirow[t]{2}{*}{ Composites } & $\begin{array}{l}\text { CERCER. Composé d'actinide + composé inerte (par exemple } \mathrm{MgO} \text { ou } \mathrm{ZrO}_{2} \text { stabiliséé à l'yttrium) } \\
\text { à l'état de céramiques } \\
\mathrm{MgO}+\mathrm{UO}_{2}(\text { à } 40 \%), \mathrm{MgO}+\mathrm{PuO}_{2}(\text { à } 60 \%), \mathrm{MgO}+\mathrm{AmO}_{2-\mathrm{x}}, \mathrm{MgO}+(\mathrm{Am} / \mathrm{Zr} / \mathrm{Y}) \mathrm{O}_{2-\mathrm{x}} \text {, } \\
(\mathrm{Pu}, \mathrm{Am}, \mathrm{Cm}) \mathrm{O}_{2-\mathrm{X}}+\mathrm{MgO}, \mathrm{TiN}+\mathrm{PuN}(\mathrm{a} 60 \%)\end{array}$ \\
\hline & $\begin{array}{l}\text { CERMET. Composé d'actinide à l'état de céramique + composé inerte métallique (par exemple } \\
\mathrm{Mo}, \mathrm{W}, \mathrm{Cr}) \\
{ }^{92} \mathrm{Mo}+(\mathrm{Am} / \mathrm{Zr} / \mathrm{Y}) \mathrm{O}_{2}\end{array}$ \\
\hline
\end{tabular}

\section{Comportement chimique probable des actinides en cas de rupture de confinement}

La rupture de confinement conduit pour le travailleur à une possible contamination interne par inhalation. C'est le cas de contamination le plus fréquent, mais qui provoque, grâce aux systèmes de détection en place et à la structure de l'arbre respiratoire, qu'à des contaminations limitées. À l'inverse, la blessure, bien que plus rare, peut conduire à des contaminations importantes. C'est par blessure que l'on a observé les contaminations les plus fortes depuis l'essor de l'industrie nucléaire. L'ingestion est très rare dans le monde du travail. En revanche, elle accompagne toujours l'inhalation, par déglutition des particules épurées par les mécanismes d'épuration pulmonaire par remontée mucociliaire. 
La rupture de confinement doit être envisagée, du point de vue de la radioprotection, aux diverses étapes de l'amont et de l'aval des réacteurs et en réacteurs, c'est-à-dire à chaque étape de la transformation de la matière nucléaire. La dissémination de produits radioactifs peut ainsi avoir lieu à différentes températures, de l'ambiante à haute température. Selon la température, la nature des produits émis peut être très différente.

Il convient de distinguer les composés volatils qui se propageant sous forme moléculaire et les composés non volatils qui se propagent sous forme d'agrégats microscopiques ou d'aérosols.

On a vu que les caractéristiques des composés solides prises en compte dans les modèles dosimétriques sont la taille, la surface spécifique, la densité des particules déposées, leur solubilité au niveau pulmonaire ou dans les fluides corporels et les espèces véhiculées dans le sang. La «spéciation» des radionucléides, au sens de leur degré d'oxydation et des espèces chimiques qu'ils forment, avant inhalation, ingestion ou blessure, joue un rôle prépondérant. C'est au regard de ces paramètres que doit être apprécié puis étudié le comportement des produits émis.

La «spéciation biologique » lorsque les radionucléides sont passés dans le sang est relativement bien connue, elle est indépendante de la forme d'entrée. Sa modélisation par la CIPR a fait l'objet de plusieurs révisions récentes. Les modèles systémiques des alcalino-terreux et du plomb d'une part, du plutonium, américium et neptunium d'autre part, ont été revisités dans la publication 67 de la CIPR (ICRP, 1994b), ceux de l'uranium et du thorium dans la publication 69 (ICRP, 1995b).

Chaque actinide (thorium à curium) a un comportement biologique spécifique qui dépend de son degré d'oxydation. Vis-à-vis de ce paramètre, les tendances sont les suivantes. Les actinides tétravalents sont peu transférables. Les oxydes sont peu solubilisés au niveau du poumon. Les aérosols qui y arrivent, sous forme de sels, sont rapidement hydrolysés et donnent des colloïdes peu solubles. Il en est de même s'il s'agit de composés moléculaires. Au niveau de la blessure, ils resteront principalement in situ. Au niveau de l'intestin, vers pH9, les actinides solubilisés dans l'estomac ( $\mathrm{pH} 1)$, sont hydrolysés et donnent aussi des colloïdes peu absorbés $\left(\mathrm{f}_{\mathrm{A}}=1 \times 10^{-4}\right)$. Dans les mêmes conditions, les oxydes et collö̈des d'actinides trivalents sont plus solubles et 10 fois mieux absorbés. L'uranium et le neptunium sont plus transférables respectivement au degré d'oxydation 6 et 5 . Ils passent mieux dans l'organisme par les poumons ou l'intestin $\left(\mathrm{f}_{\mathrm{A}}=10^{-2}\right)$ et leur comportement est finalement proche d'éléments divalents. Les actinides tétravalents vont principalement dans le squelette, les trivalents dans le foie 
(Durbin, 2006, 1973) toutefois cette répartition dépendra fortement de la stabilité des complexes qu'ils forment avec les ions citrates présents au niveau sanguin (Masse et al., 1973). Enfin l'uranium hexavalent précipite à forte concentration préférentiellement dans le rein (Diamond, 1989). Ces destinations finales sont liées à la différence de stabilité des complexes des actinides véhiculés par le sang.

Le comportement du protactinium est quasi inconnu. On ne trouve que quelques publications dans la littérature et il faut remonter à 1946 (Lanz et al., 1946), 1948 (Hamilton, 1948) pour obtenir des informations parcellaires sur les chlorures chez le rat et 1986 (Ralston et al., 1986) pour avoir des informations relatives au singe, mais après injection intraveineuse du complexe citrate, forme peu rencontrée dans les ateliers et usines.

\subsection{Combustibles et cibles autres qu'oxydes}

La nécessité de réexaminer l'emploi de matériaux autres qu'oxydes pour les réacteurs du futur vient des caractéristiques que l'on cherche pour la puissance volumique des cœurs des réacteurs, conditionnée par la densité des matériaux, leur point de fusion, leur conductibilité thermique et leur capacité à être brûlés (hauts taux de combustion). Toutefois le choix doit aussi tenir compte d'autres contraintes comme la teneur possible en actinides mineurs et en hélium, la compatibilité avec le caloporteur, la sûreté du réacteur et le retraitement du combustible usé. Le combustible métallique est très intéressant et a déjà été utilisé notamment aux USA. Cependant son point de fusion est peu élevé comparé à ceux des céramiques oxyde, nitrure ou carbure. Un système nucléaire du futur basé sur ce choix impliquerait probablement un retraitement pyrochimique. À puissance égale pour un réacteur donné, la masse de Pu par cœur est minimale pour le carbure puis viennent ensuite le nitrure et l'oxyde. Le nitrure doit être le plus possible enrichi en ${ }^{15} \mathrm{~N}$ ce qui est très pénalisant au plan énergétique. Enfin le carbure pose aussi des problèmes d'obtention de phases adéquates et de pyrophoricité. Leur dissolution pour retraitement n'est pas simple. La fabrication des assemblages de combustible à base de nitrure ou carbure n'est pas une copie de celle des assemblages à base d'oxydes. Aussi les oxydes, pour lesquels un retour d'expérience est important, pourraient-ils finalement être retenus?

Quoi qu'il en soit, du point de vue de la radioprotection et de l'évaluation du risque, il n'existe pas à notre connaissance de données radiotoxicologiques concernant les carbures et nitrures d'actinides et tout reste à faire pour avoir quelques idées des risques potentiels aux différents stades de leur préparation et de leur comportement à haute température en cas de perte de confinement. 


\subsection{Combustibles et cibles à base d'oxydes}

La préparation de MOX à partir de mélanges des dioxydes d'uranium et de plutonium est maîtrisée. Elle est industrielle. Les propriétés de ces oxydes sont suffisamment proches pour que leur frittage simultané puisse être conduit sans problème majeur au regard de la dispersion de matière radioactive. La fabrication de MOX incorporant quelques pourcents d'actinides mineurs au degré d'oxydation 4, notamment d'américium, nécessite le contrôle délicat de ce degré d'oxydation pour tous les éléments. L'américium et le curium sont essentiellement trivalents dans leur état stable et les trioxydes d'actinides sont plus « volatils » que les dioxydes. Pour éviter une émission de matière à haute température lors du frittage et pour d'autres raisons visant notamment à une bonne homogénéité microscopique de la matière nucléaire fissile, l'obtention de solutions solides des éléments est recherchée. Dans ces « solutions » les éléments perdent en partie leurs propriétés intrinsèques et les actinides mineurs trivalents y seraient moins volatils. La préparation de solutions solides est facilitée par une cogestion des actinides plutôt que par des gestions séparées. C'est ainsi que l'on recherche la co-extraction des actinides et la préparation de combustibles ou de cibles à partir de solutions renfermant tous les éléments.

Toutefois l'introduction d'actinides mineurs dans les dioxydes d'uranium et/ou de plutonium conduits souvent à l'apparition d'oxydes non-stœchiométriques, c'est-à-dire à la présence d'actinides sous deux degrés d'oxydation. On comprend dès lors que les solutions solides non-stœchiométriques pourraient avoir, du point de vue qui nous intéresse ici, des propriétés différentes des composés normaux, par exemple vis-à-vis de leur solubilité. L'apparition de composés nonstœchiométriques est déjà connue dans les MOX à haut taux de combustion où la redistribution de l'oxygène dans les aiguilles des assemblages de combustible conduit à $\mathrm{UO}_{2+\mathrm{x}}$ (U IV et VI, sur-stœchiométrie) et $\mathrm{PuO}_{2-\mathrm{x}}$ (PuIV et III, sousstœchiométrie). Ce phénomène devrait accentuer la non-stœchiométrie des nouveaux composés initialement non-stœchiométriques, appelés à subir de fortes irradiations. Ces considérations sont aussi valables pour les cibles de transmutation.

Les oxydes mixtes uranium-plutonium ont été assez bien étudiés. Les résultats dépendent fortement de la technique utilisée pour la préparation de l'oxyde. Le plutonium ne se comporte pas différemment chez le singe qu'il soit inhalé sous forme d'oxyde pur préparé à $1000^{\circ} \mathrm{C}$ ou bien sous forme d'oxyde mixte, à $20 \%$ de plutonium, préparé à $1680^{\circ} \mathrm{C}$ (Lataillade et al., 1995). Si l'oxyde mixte a été préparé à partir d'une solution solide la solubilité du plutonium, mesurée in vitro, est augmentée (Eidson et Mewhinney, 1983). Dans une autre étude Morgan et al. (1988) montrent que chez la souris, avec un oxyde mixte contenant $30 \%$ de 
plutonium et préparé à $1400{ }^{\circ} \mathrm{C}$ ou $1600{ }^{\circ} \mathrm{C}$, le transfert de cet élément vers le foie et l'os est plus élevé que pour un oxyde de plutonium pur. Plus récemment, il a été montré l'importance de la teneur en plutonium dans l'oxyde mixte au niveau de la dissolution de celui-ci (Ramounet et al., 2003). Ces résultats apparemment contradictoires, reflétant les conditions d'obtention des oxydes, montrent bien la nécessité de réaliser des études spécifiques avec des composés parfaitement caractérisés tant au plan de la taille que de la stæchiométrie.

L'épuration pulmonaire d'un mélange d'oxydes de curium, de plutonium et d'oxyde mixte de ces deux éléments a été étudiée par Rhoads et al. (1986). Les résultats montrent là encore la difficulté à prédire le comportement biologique des composés mixtes puisque le transfert du curium, habituellement rapide du poumon vers le sang est considérablement ralenti lorsqu'il est incorporé dans l'oxyde de plutonium. De plus, le transfert du plutonium sous cette forme mixte est lui aussi ralenti. Les auteurs montrent bien ainsi le rôle de la technique de préparation des oxydes mixtes dans les résultats observés, mais cela reste qualitatif.

Ainsi une mise en pratique des oxydes du futur comme combustibles ou cibles de transmutation conduit à s'interroger sur la valeur des données radiotoxicologiques dont on dispose aujourd'hui.

On sait de plus que la solubilité des oxydes de plutonium dépend, outre des conditions de préparation à différentes température qui influe certainement sur leur taille, de l'isotopie de ce radioélément (période, rayonnement émis), de son âge et de sa stœchiométrie (Bair et Park 1968; Sanders, 1972; Fleischer, 1975; Mewhinney et al., 1976 ; Fleischer et Raabe, 1978, 1977 ; Morgan et al., 1988 ; Guilmette et al., 1989 ; Chazel et al., 1998). Récemment on a compris et quantifié l'effet de la taille des oxydes tétravalents d'actinides sur leur solubilité. C'est un facteur très important. Par exemple, le produit de solubilité des oxydes de thorium peut varier considérablement en fonction de leur diamètre entre quelques nanomètres jusqu'à $100 \mathrm{~nm}$, valeur au-delà de laquelle il n'y a plus d'effet décelable (Neck et al., 2003). Les oxydes hydratés, fraîchement précipités par hydrolyse, sont en général très fins.

Qu'en sera-t-il des nouveaux oxydes, par exemple non-stœechiométriques tels que $\mathrm{MO}_{2-\mathrm{x}}$ avec $\mathrm{M}=\mathrm{Pu}, \mathrm{Am}$, (en fait $\mathrm{M}_{(1-4 \mathrm{x})}{ }^{4+} \mathrm{M}_{4 \mathrm{x}}{ }^{3+} \mathrm{O}_{2-\mathrm{x}}$ ) que l'on peut le voir formellement comme des mélanges de $\mathrm{x}_{2} \mathrm{O}_{3}$ et de (1-x) $\mathrm{MO}_{2}$. Ou bien tels que $\left(\mathrm{M}_{(1-\mathrm{x}-\mathrm{y})} \mathrm{M}_{\mathrm{x}}^{\prime} \mathrm{M}_{\mathrm{y}}^{\prime}\right) \mathrm{O}_{2-\mathrm{z}}$ (en fait $\left.\left.\mathrm{M}_{(1-\mathrm{x}-\mathrm{y})} \mathrm{M}_{(\mathrm{x}+\mathrm{y})-\mathrm{z}}^{\prime}{ }^{4+} \mathrm{M}_{\mathrm{z}}^{\prime}, 3+\right) \mathrm{O}_{2-\mathrm{z}}\right)$ un exemple étant $\left.\left(\mathrm{U}_{(1-\mathrm{x}-\mathrm{y})}\right) \mathrm{Pu}_{\mathrm{x}} \mathrm{Am}_{\mathrm{y}}\right) \mathrm{O}_{2-\mathrm{z}}$, dans lesquels le pourcentage « d'oxydes trivalents de $\mathrm{M}^{\prime}$ ' et M' indiscernables » est donné par la valeur de $\mathrm{z}$ ? Leurs solubilités, exprimées pour les différents éléments qu' ils contiendront varieront-t-elle suffisamment pour qu'ils puissent passer, selon les critères de la CIPR, de la classe $\mathrm{S}$ à $\mathrm{M}$ (facteur 
10) ? L'utilisation de facteurs par défaut tels que préconisés par la CIPR conduirat-elle à des estimations de dose efficace engagée importantes ?

On sait aussi que la «spéciation » de l'élément est importante. Qu'en sera-t-il de ce point de vue pour les espèces que donneront ces nouveaux oxydes ? Qu'en sera-t-il pour les composés intermédiaires conduisant à leur préparation ? En effet, pour préparer ces oxydes, notamment sous forme de solutions solides, il faudra emprunter de nouvelles voies, par exemple de co-précipitation des éléments à partir de solutions oxaliques renfermant les actinides sous les degrés d'oxydation 4 et 3 , toutes suivies de calcination au cours desquelles on contrôle plus ou moins bien les degrés d'oxydation. Qu'en sera-t-il au niveau du retraitement hydrochimique des combustibles et cibles de transmutation avec de nouveaux réactifs de co-extraction et de leurs produits de dégradation?

Ces interrogations montrent qu'un champ important de recherches devrait être ouvert in vitro et in vivo pour compléter les données connues et acquérir celles qui font défaut.

\subsection{Cas des sels fondus}

Les solutions d'actinides dans les sels fondus sont déjà utilisées pour traiter par voie sèche du combustible usé métallique ou oxyde afin de séparer les actinides des produits de fission. Il s'agit de solutions de chlorures ou de fluorures maintenues liquides à haute température. Selon les méthodes utilisées, les actinides y sont sous leur degré d'oxydation maximum ou minimum (OCDE, 2004). La matière fissile/fertile liquide dans le concept MSR serait un mélange de fluorures de lithium, thorium et de plutonium ou d'uranium s'enrichissant surtout en produits de fission et très peu en actinides mineurs. Un nouvel actinide apparaîtrait, le protactinium. La teneur en actinides mineurs ne serait élevée que si on souhaitait transmuter ces derniers. L'utilisation d'un combustible nucléaire liquide pose a priori de nouveaux problèmes de radioprotection en raison de l'absence de la première barrière traditionnelle de confinement. Des fluorures d'actinides pourraient se former et le retraitement in situ pour enlever les produits de fission ou récupérer l'uranium passerait par la formation de fluorures gazeux. À cet égard, on rappelle le comportement biologique particulier de $\mathrm{UF}_{4}$ qui, classé insoluble par les chimistes est très rapidement solubilisé au niveau pulmonaire (André et al., 1989a, 1989b). Les informations disponibles pour $\mathrm{PuF}_{4}$ sont contradictoires : Dilley (1970) décrit un comportement biologique après inhalation proche du nitrate de plutonium mais la même équipe le décrit comme se comportant comme un composé peu soluble similaire à l'oxyde ${ }^{239} \mathrm{PuO}_{2}$, réfractaire au traitement par le DTPA (Mc Donald et al., 1979). Il y a donc ici un 
risque de fausse prévision par analogie du comportent de $\mathrm{PuF}_{4}$ à partir de celui de $\mathrm{UF}_{4}$.

Si le devenir des halogénures d'uranium et de plutonium est plus ou moins bien connu, il l'est moins pour les halogénures de thorium et des actinides mineurs et il est inconnu pour ceux de protactinium.

\subsection{Combustion d'oxydes avec d'autres métaux}

Il faut rappeler enfin que lorsque les oxydes d'uranium et de plutonium se retrouvent accidentellement au contact de métaux, caloporteurs ou de structure, leurs propriétés chimiques peuvent être modifiés avec pour conséquence une augmentation de leur solubilité biologique (Métivier, 1976 ; Métivier et al., 1980, 1976 ; Stradling et al., 1978 ; Allen et al., 1981).

\section{Conclusion}

Il est difficile de prédire ce que seront les matières radioactives des cycles du combustible des systèmes du futur, tels que définis dans le programme "Generation IV ». Aujourd'hui les choix de compositions du combustible nucléaire sont dictés par la «fabricabilité » plus que par la recherche de performance, ceci afin de limiter les étapes des procédés où il peut y avoir dispersion de matière. Ces choix pourraient être inversés. Des tendances se font jour. Des programmes de recherches sur les combustibles et cibles de transmutation, nationaux et internationaux, ont été lancés et vont se poursuivre. On en connaît les directions. Toutefois aucun n'a réellement abordé, ou n'envisage d'aborder semble-t-il, l'étude du devenir biologique des nouvelles matières radioactives qui pourraient contaminer les individus en cas de rupture de confinement.

Il semble donc hautement nécessaire d'accompagner les études physicochimiques engagées pour les systèmes du futur par des études de radiotoxicologie. Ceci est d'autant plus urgent que l'on ne dispose d'aucune donnée pour certains des composés déjà manipulés, carbure ou nitrure de plutonium par exemple. On a également vu que le comportement biologique des actinides dans les mélanges d'oxydes ou les oxydes mixtes sont très dépendant de leur mode de préparation. La complexité des combustibles du futur étant augmentée par rapport à ces situations, il va de soit que seule l'expérimentation permettra de décrire correctement le comportement biologique des radionucléides qu'ils contiennent lors de contamination. Le médecin du travail pourra ainsi correctement estimer le risque et prescrire une thérapeutique adaptée en cas de besoin. Il déterminera 
également des protocoles après incidents et les intervalles des analyses de surveillance systématique. Cela est d'autant plus vrai, que la chimie des actinides impliqués étant particulièrement complexe, il est difficile, voire impossible, de prévoir de manière théorique le comportement biologique de ces composés, comme cela a été malheureusement expérimenté avec $\mathrm{UF}_{4}$, le complexe Pu-TBP et les différents oxydes de plutonium.

Ces études devraient concerner en premier les composés nouveaux pour lesquels l'information est inexistante, mais également les oxydes, dont la nature devient différente de celle du nucléaire actuel lorsque l'objectif est de réduire la teneur des actinides mineurs dans les déchets nucléaires. À cet égard, il faudra aborder rapidement l'étude des oxydes riches en américium et neptunium. Les études devraient comporter, comme par le passé, des études in vitro pour obtenir des données indispensables comme les tensions de vapeur des éléments dans différentes circonstances ou les espèces concernées et des études in vivo pour préciser au moins les valeurs des paramètres des modèles de contamination. Toutes ces études sont précieuses pour le médecin qui doit évaluer le risque à partir de la dose mais également de sa distribution cellulaire.

C'est par une relation de confiance entre les opérateurs, les radioprotectionnistes, radiotoxicologistes et les médecins, que l'on pourra garantir une protection d'un niveau au moins égal à celle qu'elle a été jusqu'à maintenant.

Remerciements. Les auteurs tiennent à remercier le docteur A. Miele (CEN, Cadarache) qui par ses questions est à l'origine de ce travail et Nicolas Tricot (IRSN) pour son aide dans l'examen des différents types de combustibles.

\section{RÉFÉRENCES}

Allen M.D., Briant J.K., Moss O.R., Rossignol E.J., Malhum D.D., Morgan L.G., Ryan J.L., Turcotte R.P. (1981) Dissolution characteristics of LMFBR fuel-sodium aerosols, Health Phys. 40, 183 193.

André S., Métivier H., Lantenois G., Boyer M., Nolibé D., Masse R. (1987) Beryllium metal solubility in the lung, comparison of metal and hot-pressed forms by in-vivo and in vitro dissolution bioassays, Human Toxicol. 6, 233-240.

André S., Métivier H., Auget D., Lantenois G., Boyer M., Masse R. (1989a) Lung dissolution of uranium tetrafluoride in rats and baboons. Comparison with dissolution by alveolar macrophages in culture and chemical dissolution, Human Toxicol. 8, 11-119.

André S., Métivier H., Auget D., Lantenois G., Boyer M., Masse R. (1989b) Assesment of uranium tetrafluoride dissolution in the lung by in vivo and in vitro methods, Rad. Prot. Dosim. 26, 75-81.

Ansoborlo E., Claraz M., Hengé-Napoli M.H., Métivier H., Garden J., Cheynet M.C. (1995) Etude de l'exposition industrielle à des aérosols d'uranium dans le procédé d'enrichissement par Laser Méthodes et Résultats, Radioprotection 30, 13-24.

Ansoborlo E., Chazel V., Hengé-Napoli M.H., Pihet P. Rannou A., Bailey M.R., Stradling N. (2002) Determination of the physical and chemical properties, biokinetics, and dose coefficients of uranium compounds handled huclear fuel fabrication in France, Health Phys. 82, 279-289. 
Bailey M.R., Kreyling W.G., André S., Batchelor A., Collier C.G., Drosselmeyer E., Ferron G.A., Foster P., Haider B., Hodgson A., Masse R., Métivier H., Morgan A., Müller H.L., Patrick G., Pearman I., Pickering S., Ramsden D., Strirling C., Talbot R.J. (1989) An interspecies comparison of the lung clearance of inhaled monodisperse cobalt oxide particles part I: objectives and summary results, J. Aerosol Sci. 20, 169-188.

Bair W.J., Park J.F. (1968) Comparative deposition of four types of plutonium dioxides inhaled bu dogs, In Proceedings of the 1st international congress of radiation protection, Rome, September 5-10, 1966. Pergamon Press, Oxford-New-York.

Bair W.J., Métivier H., Park J.F. (1980) Comparison of early mortality in baboons and dogs after inhalation of ${ }^{239} \mathrm{PuO}_{2}$, Radiat. Res. 82, 588-610.

Bair W.J., Park J.F., Dagle G.E., James A.C. (1989) Overview of biological consequences of exposure to plutonium and higher actinides, Rad. Prot. Dosim. 26, 125-135.

Breitenstein B.D., Durbin P.W., Goans R.E., Guilmette R., Russell J.J., Toohey R.E. (2007) The NCRP Radionuclide Dosimetry Models for Wounds, NCRP report, à paraître.

Carter M.W. (1995) Is measurement of transportability class of inhaled material by in vitro dissolution satisfactory? Health Phys. 69, 549-552.

CEA (2005) L'Energie nucléaire du futur: quelles recherches pour quels objectifs? Commissariat à l'énergie atomique, e-den, une monographie de la Direction de l'énergie nucléaire. CEA Saclay et Groupe Moniteur, Paris, ISBN 2-281-11307-8.

Chazel V., Houpert P., Ansoborlo E. (1998) Effect of $\mathrm{U}_{3} \mathrm{O}_{8}$ specific surface area on in vitro dissolution, biokinetics, and dose coefficients, Rad. Prot. Dosim. 79, 39-42.

Cooke N., Holt F.B. (1974) The solubility of some uranium compounds in simulated lung fluid, Health Phys. 27, 69-77.

Diamond G.L. (1989) Biological consequences of exposure to soluble forms of uranium, Radiat. Prot. Dosim. 26, 23-33.

Dilley J.V. (1970) Effect of DTPA on inhaled ${ }^{239} \mathrm{PuF}_{4}$ in beagles, Pacific Northwest Laboratory annual report for 1968 to the USAEC Division of biology and medicine, BNWL-1050, vol 1, part 1, p5.14-5.17.

Durbin P. (1960) Metabolic characteristics within a chemical family, Health Phys. 2, 225-238.

Durbin P. (1973) Metabolism and biological effects of the transplutonium elements, In Uranium, Plutonium Transplutonic Elements, H.C.Hodge, J.N.Stannard, J.B.Hursh (Eds). SpringerVerlag, Berlin, pp. 739-896.

Durbin (2006) Actinides in animals and man, 3339-3440.

Eidson A.F. (1994) The effect of solubility on inhaled uranium compound and clearance; a review, Health Phys. 45, 1023-1037.

Eidson A.F., Mewhinney J.A. (1983) In vitro dissolution of respirable aerosols of industrial uranium and plutonium mixed-oxide nuclear fuelss, Health Phys. 45, 1023-1037.

Fleischer R.L. (1975) On the "dissolution" of respirable $\mathrm{PuO}_{2}$ particles, Health Phys. 29, 69-73.

Fleischer R.L., Raabe O.G. (1977) Fragmentation of respirable $\mathrm{PuO}_{2}$ in water by alpha decay - A mode of dissolution, Health Phys. 32, 253-257.

Fleischer R.L., Raabe O.G. (1978) On the mechanisms of "dissolution" in liquids of $\mathrm{PuO}_{2}$ by alpha decay, Health Phys. 35, 545-548.

Gamble J.L. (1967) Chemical anatomy, Physiology and pathology of extracellular fluid, 8th edn. Harvard University Press, pp. 4-11.

GLOBAL (2003) Proceedings of GLOBAL 2003, Atoms for Prosperity: Updating Eisenhower's Global Vision for Nuclear Energy, New Orleans, Louisiana, November 16-20, 2003. ISBN 0-89448$677-2$ 
GLOBAL (2005) International conference GLOBAL 2005, Nuclear Energy Systems for future generation adn global sustainability, Tsukuba, Japan, October 9-13, 2005.

Guilmette R.A., Griffith W.C., Hickman A.W. (1989) Intake assessment for workers who have inhaled 238Pu aerosols, Rad. Prot. Dosim. 53, 127-131.

Hamilton J.G. (1948) Medical and Health Physics Quaterly Report, UCRL-41, University of Califonia, Radiation Laboratory, p. 15.

Hengé-Napoli M.H., Ansoborlo E., Donnadieu-Claraz, Berry J.P., Gibert R., Pradal B. (1994) Solubility and transferability of several industrial forms of uranium oxides, Rad. Prot. Dosim. 53, 157-161.

ICRP Publication 66 (1994a) Human respiratory tract model for radiological protection, Ann. ICRP 24(1-3).

ICRP Publication 67 (1994b) Age-dependent doses to members of the public from intake of radionuclides: Part 2 Ingestion dose coefficients, Ann. ICRP 23(3/4).

ICRP Publication 68 (1995a) Dose coefficients for intakes of radionuclides by workers, Ann. ICRP 24(4).

ICRP Publication 69 (1995b) Age-dependent doses to members of the public from intake of radionuclides: Part 3 Ingestion dose coefficients, Ann. ICRP 25(1).

ICRP Publication 100 (2007) Human alimentary tract model for radiological protection, Ann. ICRP 36(1-2).

Kanapilly G.M., Raabe O.G., Goh C.H.T., Chimenti R.H. (1973) Measurement of in vitro dissolution of aerosol particles by comparisons to in vivo dissolution in the lower respiratory tract after inhalation. Health Phys. 24, 497-507.

Lanz H., Scott K.G., Crowley J., Hamilton J.G. (1946) The metabolism of Thorium, Protactinium and Neptunim in the rat, USAEC MDDC-648 (CH-3606)

Lataillade G., Verry M., Rateau G., Métivier H., Masse R. (1995) translocation of plutonium from rat and monkey lung after inhalation of industrial plutonium oxide and mixed uranium and plutonium oxide, Int. J. Radiat Biol. 67, 3,373-380.

Lirsac P.N., Nolibé D., Métivier H. (1989) Immobilization of alveolar macrophages for measurement of in vitro dissolution of aerosols particles, Int. J. Radiat. Biol. 56, 1011-1021.

Mc Donald K.E., Dilley J.V., Sanders C.L., Mahaffey J.A. (1979) The influence of DTPA on the metabolism of inhaled ${ }^{239} \mathrm{PuF}_{4}$ in Beagles, Health Phys. 36, 632-635

Masse R., Métivier H. (1990) Intérêt du primate pour l'évaluation de la pathologie pulmonaire, Pathol. Biol. 8, 193-196.

Masse R., Métivier H., Guillaumont R. (1973) Fixation osseuse des terres rares et des éléments transuraniens. Colloques Internationaux CNRS N ${ }^{\circ} 30$, «Physico-chimie et cristallographie des apatites d'intérêt biologique », 10-15 septembre 1973, pp. 441-450.

Métivier H. (1976) Devenir biologique des mélanges d'aérosols d'oxyde de sodium et de plutonium, Radioprotection 11, 271-278

Métivier H. (1979) Devenir biologique des transuraniens inhalés, Radioprotection 14, 19-39.

Métivier H. (1989) Physico-chimie et devenir biologique des radioéléments de l'industrie nucléaire, Revue Générale Nucléaire 4, 314-317.

Métivier H., Masse R. (1986) Données nouvelles sur la biologie des actinides - Conséquences en matière de radioprotection, Journal of the Less Common Metals 122, 475-485.

Métivier H., Masse R., Nénot J.C., Nolibé D., Lafuma J. (1976) Etude expérimentale de la contamination par un mélange issu de la combustion du sodium en présence d'oxyde de plutonium, IAEA-SR-6/37, pp. 107-117.

Métivier H et al. (1980) Experimental study of respiratory contamination by a mixed oxide aerosols formed from the combustion of a plutonium magnesium alloy, Health Phys. 38, 769-776.

Métivier H., Masse R., Lafuma J. (1983) Metabolism of plutonium introduced as tri-n-butylphosphate complex in the rat and removal attempts by DTPA, Health Phys. 44, 623-634. 
Métivier H., Piechowski J., Duserre C., Rateau G., Legendre N., Menoux B., Masse R. (1989) Biological behaviour of plutonium inhaled by baboons as plutonium n-tributylphosphate complex. Comparison with ICRP models, Rad. Prot. Dosim. 26, 287-291.

Mewhinney J.A., Muggenburg B.A., McClellan R.O., Miglio J.J. (1976) The effect of varying physical and chemical characteristics of inhaled plutonium aerosols on metabolism and excretion, In Diagnosis and Treatment of Incorporated Radionuclides, IAEA, Vienna, pp. 87-97.

Morgan A., Black A., Knight D., Moores S.R. (1988) The effect of firing temperature on the lung retention and translocation of $\mathrm{Pu}$ following the inhalation of ${ }^{238} \mathrm{PuO}_{2}$ and ${ }^{239} \mathrm{PuO}_{2}$ by $\mathrm{CBA} / \mathrm{H}$ mice, Health Phys. 54, 301-310.

Moody J.C., Stradling G.N., Britcher A.R. (1994) Biokinetics of the industrial plutonium nitrate materials: implications for human exposure, Rad. Prot. Dosim. 53, 169-172.

Neck V., Altmeier M., Müller R., Bauer A., Fanghanel T., Kim J.I. (2003) Solubility of cristallin thorium dioxide, Radiochim. Acta 91, 253-262.

Nolibé D., Duserre C., Gil I., Rateau G., Métivier H. (1989) Biological behaviour of plutonium given as a trilaurylamine complex. Comparison with plutonium-tributylphosphate, Rad. Prot. Dosim. 26, 303-306.

OCDE (2004) NEA N ${ }^{\circ}$ 5427, Pyrochemical separations in nuclear applications, A status report.

OCDE (2005) NEA N ${ }^{\circ} 5419$, Fuels and materials for transmutation, A status report.

OCDE (2006) NEA $\mathrm{N}^{\circ}$ 6051, Besoins de recherche et développement pour les systèmes nucléaires actuels et futurs.

Ogawa T., Crawford D., Konings R.J.M., S. Pillon S., Schram R.P.C., Verwerft M., J. Wallenius J. (2005) State-of-art technology of fuels for burning minor actinides, An OECD/NEA study, Proceeding of Global 2005, Tsukuba, Japan, Oct. 9-13, Paper $n^{\circ} 316$.

Pillon S. (2004) ouvrage collectif dirigé par S. Pillon, Synthèse sur les combustibles et cibles de transmutation, CEA/DEN/CAD/DEC/DIR04/002.

Poncy J.L., Métivier H., Dhilly M., Verry M., Masse R. (1992) In vitro dissolution of uranium oxide by baboon alveolar macrophages, Env. Health Persp. 97, 127-130.

Ralston L.G., Cohen N., Bjattacharyya M.H., Larsen R.P., Ayres L., Oldham R.D., Moretti E.S. (1986) The metabolism and gastrointestinal absorption of neptunium and protactinium in adult baboons, in Speciation of fission and activation products in the environment, R.A. Bulman, J.R. Cooper (Eds). Elsevier, Oxford, p. 191.

Ramounet B., Rateau G., Abram M.C., Grillon G., Ansoborlo E., Bérard P., Delforge J., Fritsch P. (2003) In vivo measurement of $\mathrm{Pu}$ dissolution parameters of MOX aerosols and related uncertainties in the values of the dose per unit of intake, Rad. Prot. Dosim. 105, 153-156.

Rhoads K., Killand B.W., Mahaffey J.A., Sanders C.L. (1986) Lung clearance and translocation of ${ }^{239} \mathrm{Pu}$ and ${ }^{244} \mathrm{Cm}$ in rats following inhalation individually or as a mixed oxide, Health Phys. 51, 633-640.

Sanders C.L. (1972) Deposition patterns and the toxicity of transuranium elemenbts in lung, Health Phys. 22, 607-615.

Sato H., Yamada Y., Ishigure N., Nakano T., Enomoto H., Takahashi S., Kubota Y., Inaba J. (1999) Retention, Excretion and Translocation of ${ }^{239} \mathrm{Pu}$ in rats following inhalation of ${ }^{239} \mathrm{PuO}_{2}$ calcined at 1150 and $400^{\circ}$ C., J. Rad. Res. 40, 197-204.

Stradling G.N., Loveless B.W., Ham G.J., Smith H. (1978) the biological solubility in the rat of plutonium present in mixed plutonium-sodium aerosols, Health Phys. 35, 229-235.

Stradling G.N., Stather J.W., Gray S.A., Moody, J.C., Bailey M.R., Hodgson, A., Collier C. (1987) Studies on the metabolic behaviour of industrial actinide-bearing aerosols after deposition in the rat lung: An experimental basis for interpretating chest monitoring data and assessing limits on intake for workers, Human Toxicology 6, 365-375.

Thompson R.C. (1989) Life-span effects of ionizing radiation in the beagle dog, Pacific Northwest National Laboratory, Richland, WA 9352, pp. 31-57.

USDOE (2006) Report on basic reasearch needs for advanced nuclear energy systems, Office of basic energy sciences, USDOE, October 2006. 